Chrischta Ganz

Das Herz ist für jedes Individuum das Zentrum von Leben und Vitalität. Der Mensch erlebt das Herz als Sonne seines Systems, erfahrbar als Spender von Lebensfreude, Licht und Wärme. Was im übertragenen Sinne Liebe, Freude, Verantwortung und Grosszügigkeit bedeutet, zeigt sich im Organismus im Herz-Kreislauf-System, in der Blutzirkulation, aber auch in Wärmeprozessen und der Aufrichtekraft. Dass das Thema den Nerv der Zeit trifft, ist am enormen Ausmass an Herz-Kreislauf-Pathologien sichtbar.

\section{Referate}

Die Kongressteilnehmer erwartete ein spannendes Programm mit namhaften Referenten.

Friedemann Garvelmann, Heilpraktiker, Dozent und Autor, eröffnete den Kongress mit seinem Referat «Die humoralen Regulationsprinzipien von Herz und Gefässen». Herz und Kreislauf gehören untrennbar zusammen - wie Olaf Koob, Arzt, Dozent und Autor (Abb. 1), am zweiten Kongresstag mit seinem Referat «Warum das Herz keine Pumpe ist» ebenso betonte. Zentrales Element der Gesundheit ist, dass Funktion, Regulierung und Anpassung reibungslos zusammenspielen. Sobald der Mensch etwas von den Anpassungsprozessen spürt, ist die Regulation aus dem Gleichgewicht geraten. Welche Methode für diese Regulation gewählt wird (Heilpflanzen, Schröpfgläser, Akupunkturnadeln usw.), ist das letzte Glied der Entscheidungskette. Garvelmann be-

\title{
Erster Kongress für Traditionelle Europäische Naturheilkunde in der Schweiz
}

Zum Thema «Herz» fand am 21. und 22. November 2014 im Alten Spital in Solothurn der erste Kongress für Traditionelle Europäische Naturheilkunde (TEN) in der Schweiz statt. Der Kongress wurde vom Schweizerischen Verband der anerkannten Naturheilpraktikerinnen und Naturheilpraktiker (SVANAH) organisiert und schuf eine internationale Plattform für die Vernetzung und den Austausch über das Medizinalsystem der TEN und für die Heilmethoden, die in unserer Kultur verwurzelt sind.

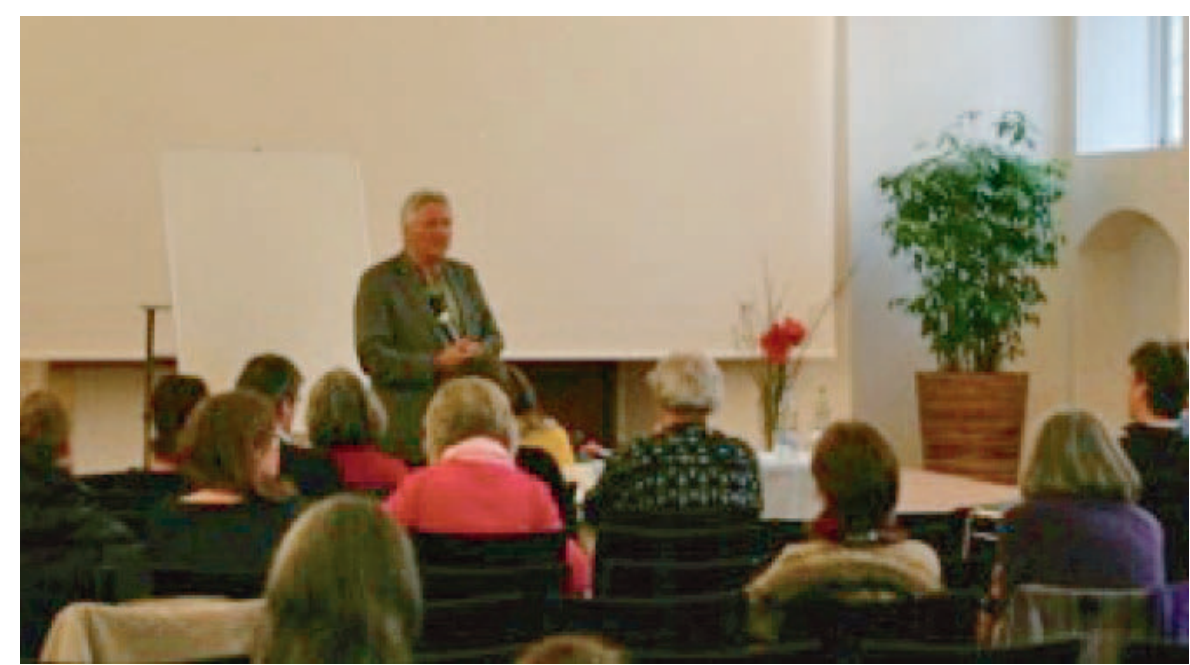

Abb. 1. Olaf Koob in seinem Referat «Warum das Herz keine Pumpe ist» (Bildquelle: TEN-Kongress).

tonte, wie wichtig das Erkennen der grundsätzlichen Mechanismen ist hyperkinetisches oder hypokinetisches Syndrom? -, bevor eine bestimmte Methode gewählt wird. Diagnose wie auch Therapie müssen auf Basis dieser grundsätzlichen Überlegungen gewählt werden - und nicht umgekehrt. Denn: Das Denken in Indikationen oder Therapiemethoden kann den Menschen nicht als Individuum erfassen.

Die humoralen Qualitäten des Blutes haben eine entscheidende Bedeutung für die Dynamik und die Anpas- sung des Herz-Kreislauf-Systems an die wechselnden Bedingungen des Lebens. Kein Organsystem reagiert so rasch mit hyper- bzw. hypokinetischen Symptomen auf Veränderungen der Blutqualität wie die Organe, die direkt mit dem Blut in Verbindung stehen. Das Wärmeprinzip steht an erster Stelle, denn die physiologische Wärme des Blutes ist der wichtigste herz- und gefässdynamisierende Faktor. Hufeland bezeichnete sie als «Reizkraft des Herzens».

Pathologische Wärme (in der TEN die sogenannten «gelbgalligen Schär-

\section{KARGER}

() 2015 S. Karger GmbH, Freiburg
Chrischta Ganz 
fen») hingegen schwächt das HerzKreislauf-System. Werden HerzKreislauf-Erkrankungen unter diesen Gesichtspunkten betrachtet, wird das Zusammenspiel mit anderen Organsystemen sichtbar. Hypokinetische Prozesse zeigen sich aufgrund mangelnder Wärmekraft. Mögliche Ursachen dafür sind fehlende physiologische Wärme, übermässige Feuchtigkeit und sogenannte "melancholische Schärfen», die sich im Menschen in kalter und trockener Qualität zeigen, was sich mittels Stagnation und chronischen Krankheitsprozessen äussern kann. Sind melancholische Schärfen die Grundlage der Dysbalance, erhält die Milz, das vergessene Organ, ihre grosse Bedeutung zurück.

Mit diesem Startreferat legte Garvelmann auf eindrückliche Weise das Fundament des Kongresses: Dass das systemische Denken des Medizinsystems der TEN und demnach jeder therapeutische Gedankengang und jeder Therapieschritt auf dem Erkennen der Zusammenhänge basieren muss.

Christian Raimann, Naturheilpraktiker, Dozent und Autor, zeigte in seinem Workshop «Die humoralen Qualitäten von Herz-Kreislauf-Pflanzen» die Sichtweise der TEN in der Pflanzenheilkunde auf. Während in der Phytotherapie (im Speziellen in der rationalen Phytotherapie) die Wirkstoffe einer Pflanze im Vordergrund stehen, erhält die TEN ihre Kenntnis über die Wirkprinzipien der Heilpflanzen. Auch hier wird die ganzheitliche Betrachtung des Menschen erkennbar.

Louis Hutter, Naturheilpraktiker und Dozent, beleuchtete «die Behandlung von Mikrozirkulationsstörungen» aus der Sicht der Gemmotherapie. Die Nutzung pflanzlichen Embryonalgewebes wurde im vorigen Jahrhundert von Dr. Henry Pol (1918-1988) als Phytoembryotherapie entwickelt und ist heute als Gemmotherapie bekannt. Die wichtigsten Gemmomazerate für die Behandlung von Mikrozirkulationsstörungen sind Cornus sanguinea (Roter Hartriegel), Alnus glutinosa

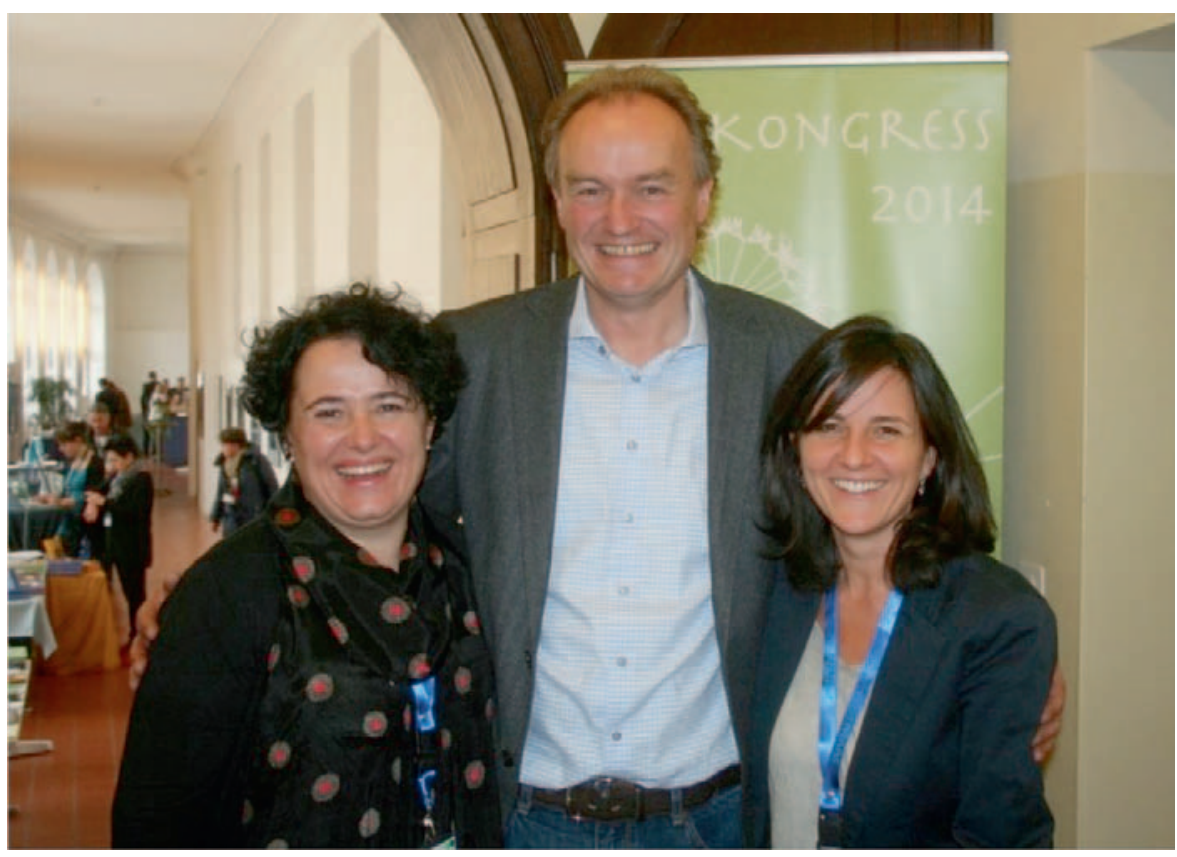

Abb. 2. Die Organisatorinnen des TEN-Kongresses Rosmarie Fehr-Streule (links) und Claudia Cairone (rechts) mit dem Referenten Olaf Rippe (Mitte) (Bildquelle: TEN-Kongress).

(Schwarz-/Roterle), Corylus avellana (Hasel), Crataegus oxyacantha (Weissdorn), Ginkgo biloba (Ginkgo/Tempelbaum), Olea europea (Ölbaum) und Rosmarinus officinalis (Rosmarin).

Conwitha Lapke, Biochemikerin und Spagyrikerin, warf in ihrem Vortrag "Herz und Herzpflanzen aus Sicht der Spagyrik» einen Blick auf die dem Leben zugrunde liegenden Prinzipien aus der Alchemie. Gemäss dem Hermetischen Gesetz «Wie aussen, so innen» oder «Wie oben, so unten» weist die anatomische, physiologische und biochemische Betrachtung des Herz-Kreislauf-Systems auf das Wirken dieser Lebensprinzipien. Mit Sicht auf die Evolution des Herzens betonte Lapke die enorme Bedeutung des Gefässsystems.

Nach Stefan von Löwensprung, anthroposophischer Arzt, Autor und heute in der medizinisch-wissenschaftlichen Abteilung der Firma Weleda tätig, spielen «Metalle in der Therapie» eine zentrale Rolle. In seinem Referat "Gold in integrativen Gesamtkonzepten der Anthroposophischen Medizin für das Herz-Kreislauf-System» zeigte er die durch Paracelsus vertretene traditionelle Heilkunst des Abendlandes auf und bezeichnete die Metalle als Vermittler zwischen den Organen im menschlichen Organismus und den kosmischen Kräften der Planeten. Die Sonne gilt als wirksames Prinzip auf das Herz-Kreislauf-System, denn das Herz ist die Sonne im Sonnensystem Mensch.

Olaf Rippe, Naturheilpraktiker, Autor und Dozent (Abb. 2), nahm den Faden des Paracelsus in seinem Referat «Naturverehrung und Heilkunst die spirituellen Grundlagen des Heilens nach Paracelsus» wieder auf. $\mathrm{Pa}$ racelsus, einer der bedeutendsten Ärzte der abendländischen Medizin, verband Volks- und Erfahrungsmedizin mit der damaligen universitären Medizin und integrierte christliche Mystik, Astrologie, Alchemie und magische Wahrnehmung der Naturkräfte. Mit Paracelsus' Aussage «Die Liebe ist die höchste Medizin» rundete Rippe sein Referat ab.

Jim M. Müller, Naturheilpraktiker und Dozent, zeigte in seinen Ausführungen zum Thema «Diagnostik von Herz-Kreislauf in der TEN» die komplexe funktionelle Einheit von Herz, Gefässsystem und Blut sowie deren ge- 
meinsame Regulationsmechanismen, die mit den diagnostischen Methoden der TEN erkennbar werden.

Verändern sich die humoralen Qualitäten in unphysiologischer Weise, resultieren daraus sowohl Dysregulationen in der Gefässmotorik als auch pathologische Abweichungen in der Herzdynamik, die mit Iris-, Zungen-, Puls- und Harndiagnostik wie auch mittels Reflexzonen erkannt werden und ein umfassenderes Bild ergeben als der Name eines Symptoms.

Cornelia Stern, Apothekerin, Dozentin und Autorin, erläuterte in ihrem Vortrag «Herz unter Druck - Trifloris-Essenzen für bedrückte Menschen», wie sehr «Herzensangelegenheiten» unmittelbar und existenziell auf das Herz wirken.

Martin Keymer, Naturheilpraktiker und Autor, griff das Thema «Psychoneuroendokrinoimmunologie» auf und betonte, wie wichtig das emotionale, bioenergetische und biophysikalische Zusammenspiel für die Regulation ist.
Im Weiteren zeigte Stefan Schaub, Heilpraktiker, Dozent und Autor, naturheilkundliche Behandlungsmethoden bei Hypertonie auf.

Vreni Brumm, diplomierte Pflegefachfrau HF und Autorin, stellte in ihrem Workshop «Wickel und Kompressen - Kraft für das Herz» konkrete Anwendungen vor, die bei Stress, Unruhe und vegetativen Störungen des Herzens angewendet werden können.

Monique Stahlkopf stellte das Herz in ihrem Referat «Das Herz - ein ausgleichendes Organ» als denkendes und fühlendes Organ vor, das sehr schnell auf Veränderungen des Gemüts reagiert - gerade auch in Bezug auf die Vorstellung, dass Krankheit «ein Heilbestreben des Organismus» ist.

Sabine Haag, Köchin, Dozentin und Autorin, leitete eine Austauschund Diskussionsrunde zum Thema «Ernährung» und ging der Frage nach, wie weit ein exakter Ernährungsplan nach Nährwerttabelle eine gesunde Ernährung bei Herz-Kreislauf-Erkrankungen ermöglicht.
René Schwarz leitete im Anschluss die Diskussion «Was ist Naturheilkunde?».

Auch die Situation in der Schweizerischen Berufspolitik der Naturheilpraktiker und Naturheilpraktikerinnen war Teil des Kongresses. Rudolf Happle berichtete über «Aktuelles von der HFP (Höhere Fachprüfung)», Remo la Marra referierte über «die HFP aus der Sicht eines praktizierenden Naturheilpraktikers», und Sybille Binder stellte die "IG TEN (Interessengemeinschaft Traditionelle Europäische Naturheilkunde)» vor.

\section{Ausblick}

Nach diesem ausserordentlich spannenden Kongress lohnt sich der Ausblick auf den zweiten TEN-Kongress am 17./18. November 2016: Dieser wird - unter anderem mit renommierten Referentinnen wie Heide Fischer und Susanne Römer - sich mit dem Thema «Frauenheilkunde» befassen. 